\title{
Three-year results of a randomized controlled trial comparing mechanochemical and thermal ablation in the treatment of insufficient great saphenous veins
}

\section{Vähäaho, Sari}

2021-05

Vähäaho , S , Halmesmäki , K, Mahmoud, O , Albäck , A, Noronen , K \& Venermo , M 2021 , ' Three-year results of a randomized controlled trial comparing mechanochemical and thermal ablation in the treatment of insufficient great saphenous veins ' , Journal of vascular surgery: Venous and lymphatic disorders, vol. 9 , no. 3 , pp. 652-659 . https://doi.org/10.1016/j.jvsv.2020.08.007

http://hdl.handle.net/10138/333075

https://doi.org/10.1016/j.jvsv.2020.08.007

cc_by_nc_nd

acceptedVersion

Downloaded from Helda, University of Helsinki institutional repository.

This is an electronic reprint of the original article.

This reprint may differ from the original in pagination and typographic detail.

Please cite the original version. 
Three-year results of a randomized controlled trial comparing mechanochemical and thermal ablation in the treatment of insufficient great saphenous veins

S. Vähäaho ${ }^{1}$, K. Halmesmäki ${ }^{1}$, O. Mahmoud ${ }^{1,}$ A. Albäck ${ }^{1}$, K. Noronen ${ }^{1}$, and M. Venermo ${ }^{1}$

${ }^{1}$ Department of Vascular Surgery, Helsinki University Hospital, Helsinki University, Helsinki, Finland

Original article

Correspondence to: Dr S. Vähäaho, Department of Vascular Surgery, Helsinki University Hospital, Helsinki University, PL 340, 00029 HUS, Helsinki, Finland (e-mail: sari.vahaaho@ fimnet.fi)

Clinicaltrials.gov registration number NCT03722134

Key words

Randomized controlled trial, venous insufficiency, varicose veins, radiofrequency ablation, laser ablation 


\section{ARTICLE HIGHLIGHTS}

Type of Research: single-center randomized controlled study

Key Findings: The occlusion rate for MOCA at three years after GSV ablation is lower (82\%) than for EVLA or RFA (both $100 \%$ ). Veins with diameters of over $7 \mathrm{~mm}$ recanalized more often than those with smaller diameter.

Take home Message: Though MOCA has some advantages over thermal ablation, the occlusion rate remains inferior at mid-term follow-up.

\section{Table of Contents Summary}

This randomized controlled study compares MOCA against thermal ablation in GSV insufficiency. At three years, the occlusion rates for MOCA are inferior to thermal ablation and it seems that veins with larger preoperative diameters have a tendency to recanalize after MOCA. 


\section{Abstract}

\section{Objectives}

Mechanochemical ablation (MOCA) is a non-thermal non-tumescent method of treating saphenous vein insufficiency. The feasibility and short-term results of MOCA are good, but its long-term results are unknown. A randomized study was performed to compare MOCA with endovenous laser ablation (EVLA) and radiofrequency ablation (RFA) in the setting of unilateral GSV insufficiency.

\section{Methods}

Venous outpatient clinic patients with varicose veins (CEAP class C2-4) caused by GSV insufficiency were invited to participate in the study; in total, 132 patients filled the inclusion criteria and were willing to participate. Patients were randomized to treatment $(2: 1: 1$ for MOCA, EVLA, and RFA, respectively). The state of the GSV with duplex Doppler ultrasound examination and the disease-specific quality of life were assessed at one month, one year, and three years after the treatment.

\section{Results}

Some patients declined to continue in the study after randomization; in total, 117 patients underwent treatment. At three years, the occlusion rate was significantly lower with MOCA than with either EVLA or RFA ( $82 \%$ vs. $100 \%, P=0.005$ ). Quality of life was similar between the groups. In the MOCA group, GSV's that were preoperatively over $7 \mathrm{~mm}$ in diameter were more likely to recanalize during the follow-up period. The partial recanalizations of proximal GSV observed at one year progressed during the follow-up.

\section{Conclusions}


MOCA is a feasible treatment option for outpatient setting, but its technical success rates are inferior compared to endovenous thermal ablation. Its use in large calibre veins should be carefully considered. 


\section{Introduction}

In the treatment of superficial venous insufficiency, there has been a paradigm shift in the last decades. Open surgery has been all but abandoned in favour of minimally invasive endovenous techniques such as endovenous laser ablation (EVLA) and radiofrequency ablation (RFA). They have long-term occlusion rates of $88 \%$ or more in randomized controlled trials, confirming that they are at least equal to high ligation and stripping ${ }^{1-5}$. Thermal ablation (EVLA and RFA) is currently recommended as first choice of treatment for saphenous vein incompetence ${ }^{6}$. These techniques, however, require the use of tumescent anaesthesia and carry the risk of thermal injury to adjacent tissues. New, non-thermal non-tumescent techniques have been developed. These include mechanochemical ablation (MOCA) and cyanoacrylate closure. The safety and short-term efficacy of MOCA has been demonstrated ${ }^{7-13}$; however, data on longer-term results are insufficient ${ }^{14-16}$. We conducted a study comparing MOCA with EVLA and RFA in the treatment of great saphenous vein (GSV) insufficiency in a randomized setting.The one-year results have been published earlier, finding that MOCA produced a lower occlusion rate than EVLA or RFA ${ }^{17}$. Here presented are the three-year follow-up results of that study.

\section{Methods}

The study protocol and methods have been described in detail previously ${ }^{17}$. Briefly, in the years 2014-2015, all patients referred to a vascular surgery clinic for treatment of varicose veins were screened for eligibility in this study, and those meeting the inclusion criteria were invited to participate. The inclusion criteria comprised clinical classification $\mathrm{C} 2-\mathrm{C} 4$, ultrasound-verified reflux of GSV in one leg with the mean GSV diameter in the thigh between 5 and 12 millimetres and age 20-75 years. Patients with BMI over $40 \mathrm{~kg} / \mathrm{m} 2$, peripheral arterial disease, lymphoedema, 
pregnancy, allergy to either lidocaine or the sclerosant, severe general illness, previous varicose vein intervention in the same leg, or coagulation disorders were excluded. The study design was approved by the Ethics Committee of Helsinki University Hospital, and all participants provided written informed consent beforehand according to the principles of the Declaration of Helsinki.

The primary outcome was the occlusion rate of the treated GSV at three years. The GSV was considered occluded, if no recanalized sections were observed in the ultrasound examination. Secondary outcomes consisted of disease-specific quality of life, clinical results measured with Venous Clinical Severity Score (VCSS) ${ }^{18}$, the need for additional treatment of the superficial veins in the same leg, and complications.

\section{Randomization}

A study nurse randomized the patients with the method of block randomization with sealed envelopes. The patients were randomized to three groups: EVLA, RFA, and MOCA in the ratio of $1: 1: 2$; the surgeon and the patient were not blinded to the treatment group.

\section{Procedures}

The procedures have been described in detail in the one-year follow-up report ${ }^{17}$. Briefly, all patients were treated in a day-surgery unit. All procedures were done under ultrasound guidance. Thermal ablation procedures (EVLA and RFA) were done under local tumescent anaesthesia, and the patients received a light sedative (diazepam) before and during (propofol) the treatment, if necessary. In thermal ablation, the thigh part (from just below the knee to the groin) of the GSV was treated. The laser fibre used for EVLA was a 1470-nm diode radial laser (ELVes ${ }^{\circledR}$; Biolitec, Bonn, Germany) VNUS Closure FAST ${ }^{\mathrm{TM}}$ catheter (VNUS Medical Technologies, San Jose, California, 
USA) was used in the RFA procedure. After thermal ablation, local phlebectomies were done under local anaesthesia through small skin incisions. In the MOCA group, ClariVein ${ }^{\circledR}$ catheter (Vascular Insights, Madison, Connecticut, USA) was used to induce occlusion in the GSV, accessing GSV at just below the knee, and liquid sodium tetradecyl sulphate (1.5\%) was used as the sclerosant. After MOCA procedure, stab wound phlebectomies were done similarly to EVLA and RFA groups, with the aftercare being the same for all three treatment groups: a support stocking for two days continuously and after that for five days during daytime.

\section{Assessments}

At baseline, the patients completed the Aberdeen Varicose Veins Questionnaire (AVVQ) ${ }^{19}$. The mean diameter of the GSV was documented before treatment both, at the proximal $20 \mathrm{~cm}$ and at the thigh. The clinical status of the venous disease was recorded with VCSS.

At one- and three-year follow-up visits, the status of the GSV was investigated using duplex Doppler ultrasound imaging. All patients were examined by the same ultrasound qualified nurse. If at least $5 \mathrm{~cm}$ of compressible, patent vein was observed, the vein segment was considered to be partially recanalized. Any additional varicosity treatment received during the follow-up was noted, as well as the need for such treatments. Nerve injuries, pigmentation, and clinical status (VCSS) was recorded. Patients also completed the AVVQ.

\section{Statistical analysis}

According to sample size calculations, to detect a 20 per cent difference in GSV occlusion rate between the treatment groups with $\alpha=0.05$ and $\beta=0 \cdot 8$, the study would require 160 patients, accounting for 5 per cent of loss during the follow-up period.The data analysis was performed 
according to intention-to-treat principle. One-way independent ANOVA was used for continuous variables with a normal distribution; when the distribution was skewed, Kruskal-Wallis test was applied. Comparison of categorical values was carried out with $\chi^{2}$ test, and the correlation between recanalization rate and the preoperative diameter of the GSV was calculated by binary logistic regression. Statistical analysis was done using $\operatorname{SPSS}^{\circledR}$ for Windows ${ }^{\circledR}$ versions 22.0-25.0 (IBM, Armonk, New York, USA).

\section{Results}

In total, 125 patients met the inclusion criteria and received the allocated intervention. 117 patients attended the one-year follow-up, and $106(84.8 \%)$ the three-year follow-up (figure 1). The patient attributes at the beginning of the study were similar between the treatment groups (table 1). There was one cross-over from MOCA to EVLA, all other patients received the allocated treatment. Phlebectomies were performed on $100 \%$ of the patients in the MOCA group, $94 \%$ of the patients in the EVLA group and $97 \%$ of the patients in the RFA group.

\section{Primary outcome}

At the three-year follow-up, all patients treated with either EVLA or RFA had a completely occluded GSV, with no observed partial or total recanalization; the occlusion rate in these groups was $100 \%$. In the MOCA group 41 of 50 GSV's were completely occluded, yielding an occlusion rate of 82 per cent $(\chi 2=11,015, P=0,005)$ (figures $2 A-B)$. However, one patient in the MOCA group had already had re-treatment with EVLA at three years; the unassisted occlusion rate for MOCA is therefore 80 per cent. Kaplan-Meier estimate yielded a significant difference between the groups; $P=0.002$ (figure 3). 
In the MOCA group, the preoperative diameter of the most proximal $20 \mathrm{~cm}$ of the GSV was strongly associated with recanalization of this segment at three years (odds ratio $1.95,95 \%$ c.i. 1.09$3.49, P=0.024$ ); odds of recanalization increased when the diameter increased (figure 4). Similarly, the diameter of the thigh trunk GSV correlated with recanalization of the trunk at three years (odds ratio $2.15,95 \%$ c.i. $1.15-4.00, P=0.016)$. Veins that had a preoperative diameter of over 7 millimetres were more likely to recanalize during the follow-up period (figure 5): the occlusion rate was $100 \%$ for veins with a diameter of circa $6 \mathrm{~mm}, 87.5 \%$ for $7 \mathrm{~mm}$ and $75 \%$ for $8 \mathrm{~mm}$.

The partial recanalization observed in the proximal GSV at one-year follow-up led to further recanalization of the GSV trunk at three years (table 2)).

\section{Secondary outcomes}

Disease-specific quality of life was measured with AVVQ and the clinical status with the VCSS. There were no differences between the groups at the baseline, and at three years, the quality of life had increased in all groups. Likewise, the clinical status had improved, with no significant differences between the treatment groups (figures 6-7).

In the MOCA group, two patients had received additional treatment during the three-year followup: one had laser ablation of the GSV (as mentioned above), and one sclerotherapy for GSV branch varicosities. At the point of three-year follow-up, two MOCA patients were in the queue for laser ablation of the GSV, and a few patients waited for consultation on their possible additional treatment. None of the patients in the EVLA and RFA groups had received additional treatment, but two in the RFA group were scheduled for treatment for branch varicosities. 
At three years, there were no patients in the MOCA group with symptoms of nerve injury. In the EVLA group, two patients had a sensory disturbance of an area under $10 x 10 \mathrm{~cm}$, one of them being in the ankle and other in the shin. In the RFA group, one patient had a small area of sensory disturbance in the thigh. All of these were present at one-year follow-up visit. One patient in the EVLA group reported a sensory disturbance of the medial ankle at one year, but this was no longer present at three years.

\section{Discussion}

In the present paper we report the three-year results of a randomized controlled trial comparing mechanochemical GSV ablation with thermal ablation. GSV occlusion rate at 3 years was significantly better after thermal ablation than after MOCA, and a clear association of GSV calibre and recanalization was noted. In a recent meta-analysis, MOCA was equal to thermal ablation in the short term; the results of this study bring valuable information about the long-term effectiveness of MOCA $^{16}$.

The unassisted 3-year occlusion rate of the GSV was $80 \%$ in the MOCA group, a slightly lower figure than in most previous reports ${ }^{8,10,11}$. However, the quality of life measured with diseasespecific instrument was equal between the three treatment groups. The study by Holewijn et al. likewise reports no significant differences in the quality of life between MOCA and other endovenous ablation methods a few years after the treatment ${ }^{10}$.

A recent publication of the two-year results of a study comparing MOCA with RFA reports a closure rate of $80.0 \%$ after MOCA and hypothesizes that using polidocanol instead of STS might lead to more recanalization, and that treating the most proximal section on the GSV twice in case it 
hasn't collapsed after the first cycle might help prevent recanalization ${ }^{10}$. In the present study, STS was used in all patients, and according to the manufacturer's instructions for use, the proximal section was treated twice if necessary. Still, the results are similar.

According to these results, partial recanalization of the proximal GSV at one year often leads to full recanalization of either the whole or proximal GSV in the following two years. This indicates that partial recanalization after MOCA is important, even if asymptomatic, since it precludes complete recanalization and may result in need of additional treatment. Recanalization seems to be more likely in the larger veins with preoperative diameter of over 7 millimetres. There are no guidelines on the maximum vein size in MOCA, but according to a recent review, Clarivein ${ }^{\circledR}$ catheter can be used in veins with diameter of up to $20-24 \mathrm{~mm}$ : the rotating tip can in such veins still reach the vein wall $^{20}$. The observed recanalization rate and the tendency of large veins to recanalize is comparable to the one-year results of this study ${ }^{17}$. Complete occlusion rate (with no recanalized areas of over 5 $\mathrm{cm})$ was not different at three years: $82 \%$ at one year, and $80 \%$ at three years.

The advantage of MOCA is that it needs no tumescence, and the risk of nerve injury is minimal. In this study, some patients in the thermal ablation group reported a local sensory deficit at one year after the procedure. In this study, at three years, most of these patients still had the deficit, although the affected areas had generally diminished in size. MOCA is a faster method than thermal ablation and might be more feasible to use in the outpatient setting. Reportedly the pain scores and swelling are lower during and after the procedure than with thermal ablation ${ }^{10,21,22}$.

This study is limited in that the inclusion criteria were very strict and therefore the patients included may not represent the real-life scope of patients requiring treatment for superficial venous insufficiency. Also, the patients and surgeons could not be blinded to the operation. The 
concomitantly performed hook phlebectomies affect the pain scores and might also contribute to nerve injuries. There was one cross-over from MOCA to EVLA, but the analysis was made according to intention-to-treat principle; the treated vein remained occluded all through the study, and therefore it doesn't lower the occlusion rate calculated for MOCA. The recruitment for the study was stopped before the target sample size was acquired, and thus the study may be underpowered. Although the attendance rate at three years was acceptable, the absolute of number of patients in each group was small and most of the treated veins had a preoperative diameter of 5 to 7 millimetres. Our results suggest that veins with a diameter of over $7 \mathrm{~mm}$ tend to recanalize more often, but data from larger patient groups are needed to confirm the results before a definite recommendation on the maximum size can be made.

We conclude that the three-year occlusion rates of MOCA are inferior compared to RFA or EVLA. Recanalization seems to be more common in veins with a preoperative diameter of over 7 millimetres, and thus MOCA should be used with due consideration in large veins. 
Acknowledgements

The authors would like to thank the study nurses for making this study possible, as well as the vascular surgeons and staff in Helsinki University Hospital.

Funding

This research did not receive any specific grant from funding agencies in the public, commercial, or not-for-profit sectors. 


\section{References}

1. van der Velden, S. K., Biemans AA, De Maeseneer MG, et al. Five-year results of a randomized clinical trial of conventional surgery, endovenous laser ablation and ultrasound-guided foam sclerotherapy in patients with great saphenous varicose veins. Br J Surg. 2015;102(10):1184-1194.

2. Vahaaho S, Halmesmaki K, Alback A, Saarinen E, Venermo M. Five-year follow-up of a randomized clinical trial comparing open surgery, foam sclerotherapy and endovenous laser ablation for great saphenous varicose veins. Br J Surg. 2018;105(6):686-691. doi: 10.1002/bjs.10757 [doi].

3. Wallace T, El-Sheikha J, Nandhra S, Leung C, Mohamed A, Harwood A, et al. Long-term outcomes of endovenous laser ablation and conventional surgery for great saphenous varicose veins. Br J Surg. 2018;105(13):1759-1767. doi: 10.1002/bjs.10961 [doi].

4. Flessenkamper I, Hartmann M, Hartmann K, Stenger D, Roll S. Endovenous laser ablation with and without high ligation compared to high ligation and stripping for treatment of great saphenous varicose veins: Results of a multicentre randomised controlled trial with up to 6 years follow-up. Phlebology. 2016;31(1):23-33. doi: 10.1177/0268355514555547 [doi].

5. Lawaetz M, Serup J, Lawaetz B, Bjoern L, Blemings A, Eklof B, et al. Comparison of endovenous ablation techniques, foam sclerotherapy and surgical stripping for great saphenous varicose veins. extended 5-year follow-up of a RCT. Int Angiol. 2017;36(3):281-288. doi: 10.23736/S0392-9590.17.03827-5 [doi].

6. Bozkurt AK, Lawaetz M, Danielsson G, Lazaris AM, Pavlovic M, Olariu S, et al. European college of phlebology guideline for truncal ablation. Phlebology. 2019:268355519857362. doi: $10.1177 / 0268355519857362$ [doi].

7. Deijen CL, Schreve MA, Bosma J, de Nie AJ, Leijdekkers VJ, van den Akker PJ, et al. Clarivein mechanochemical ablation of the great and small saphenous vein: Early treatment outcomes of two hospitals. Phlebology. 2016;31(3):192-197. doi: 10.1177/0268355515600573 [doi]. 
8. Kim PS, Bishawi M, Draughn D, Boter M, Gould C, Koziarski J, et al. Mechanochemical ablation for symptomatic great saphenous vein reflux: A two-year follow-up. Phlebology. 2017;32(1):43-48. doi: $10.1177 / 0268355515627260$ [doi].

9. Lane TR, Bootun R, Dharmarajah B, Lim CS, Najem M, Renton S, et al. A multi-centre randomised controlled trial comparing radiofrequency and mechanical occlusion chemically assisted ablation of varicose veins - final results of the venefit versus clarivein for varicose veins trial. Phlebology. 2017;32(2):89-98. doi: $10.1177 / 0268355516651026$ [doi].

10. Holewijn S, van Eekeren, R. R. J. P., Vahl A, de Vries, J. P. P. M., Reijnen, M. M. P. J., MARADONA study group. Two-year results of a multicenter randomized controlled trial comparing mechanochemical endovenous ablation to RADiOfrequeNcy ablation in the treatment of primary great saphenous vein incompetence (MARADONA trial). J Vasc Surg Venous Lymphat Disord. 2019;7(3):364-374. doi: S2213333X(19)30132-5 [pii].

11. Witte ME, Holewijn S, van Eekeren RR, de Vries JP, Zeebregts CJ, Reijnen MM. Midterm outcome of mechanochemical endovenous ablation for the treatment of great saphenous vein insufficiency. $J$ Endovasc Ther. 2017;24(1):149-155. doi: 10.1177/1526602816674455 [doi].

12. Boersma D, van Eekeren RR, Werson DA, van der Waal, R. I., Reijnen MM, de Vries JP. Mechanochemical endovenous ablation of small saphenous vein insufficiency using the ClariVein((R)) device: One-year results of a prospective series. Eur J Vasc Endovasc Surg. 2013;45(3):299-303. doi: 10.1016/j.ejvs.2012.12.004 [doi].

13. Tang TY, Kam JW, Gaunt ME. ClariVein(R) - early results from a large single-centre series of mechanochemical endovenous ablation for varicose veins. Phlebology. 2017;32(1):6-12. doi: 10.1177/0268355516630154 [doi]. 
14. Witte ME, Zeebregts CJ, de Borst GJ, Reijnen, M. M. P. J., Boersma D. Mechanochemical endovenous ablation of saphenous veins using the ClariVein: A systematic review. Phlebology. 2017;32(10):649-657. doi: $10.1177 / 0268355517702068$ [doi].

15. Sun JJ, Chowdhury MM, Sadat U, Hayes PD, Tang TY. Mechanochemical ablation for treatment of truncal venous insufficiency: A review of the current literature. J Vasc Interv Radiol. 2017;28(10):14221431. doi: S1051-0443(17)30657-7 [pii].

16. Hassanin A, Aherne TM, Greene G, Boyle E, Egan B, Tierney S, et al. A systematic review and metaanalysis of comparative studies comparing nonthermal versus thermal endovenous ablation in superficial venous incompetence. J Vasc Surg Venous Lymphat Disord. 2019;7(6):902-913.e3. doi: S2213333X(19)30352-X [pii].

17. Vähäaho S, Mahmoud O, Halmesmäki K, Albäck A, Noronen K, Vikatmaa P, et al. Randomized clinical trial of mechanochemical and endovenous thermal ablation of great saphenous varicose veins. Br J Surg. 2019;106(5):548-554. Accessed Apr 1, 2019. doi: 10.1002/bjs.11158.

18. Rutherford RB, Padberg FT,Jr, Comerota AJ, Kistner RL, Meissner MH, Moneta GL. Venous severity scoring: An adjunct to venous outcome assessment. J Vasc Surg. 2000;31(6):1307-1312. doi: S07415214(00)90143-5 [pii].

19. Garratt AM, Macdonald LM, Ruta DA, Russell IT, Buckingham JK, Krukowski ZH. Towards measurement of outcome for patients with varicose veins. Qual Health Care. 1993;2(1):5-10. doi: 10.1136/qshc.2.1.5 [doi].

20. Belramman A, Bootun R, Onida S, Davies AH, Lane TRA. ClariVein®, mechanochemical endovenous ablation: Patient selection and perspective. Journal of Vascular Diagnostics and Interventions. 2019;7:1-8. https://doi.org/10.2147/JVD.S167491.

21. Bootun R, Lane TR, Dharmarajah B, Lim CS, Naejm M, Renton S, et al. Intra-procedural pain score in a randomised controlled trial comparing mechanochemical ablation to radiofrequency ablation: The 
multicentre venefit versus ClariVein(R) for varicose veins trial. Phlebology. 2016;31(1):61-65. doi: $10.1177 / 0268355514551085$ [doi].

22. van Eekeren RR, Boersma D, Holewijn S, Werson DA, de Vries JP, Reijnen MM. Mechanochemical endovenous ablation for the treatment of great saphenous vein insufficiency. J Vasc Surg Venous Lymphat Disord. 2014;2(3):282-288. 